\title{
Modern intraoperative imaging modalities for the vascular neurosurgeon treating intracerebral hemorrhage
}

\author{
Oded Goren, M.D., ${ }^{1}$ Stephen J. Monteith, M.D., ${ }^{3}$ Moshe Hadani, M.D., ${ }^{1}$ \\ Mati BaKon, M.D., ${ }^{2}$ and SAGi HaRnof, M.D. ${ }^{1}$ \\ ${ }^{1}$ Department of Neurosurgery and the Neurovascular Unit, and ${ }^{2}$ Department of Radiology, The Chaim Sheba \\ Medical Center, Tel Hashomer, and The Sackler School of Medicine, Tel Aviv University, Tel Aviv, Israel; and \\ ${ }^{3}$ Department of Neurological Surgery, University of Virginia Health System, Charlottesville, Virginia
}

\begin{abstract}
This paper reviews the current intraoperative imaging tools that are available to assist neurosurgeons in the treatment of intracerebral hemorrhage (ICH). This review shares the authors' experience with each modality and discusses the advantages, potential limitations, and disadvantages of each.

Surgery for ICH is directed at blood clot removal, reduction of intracranial pressure, and minimization of secondary damage associated with hematoma breakdown products. For effective occlusion and safe obliteration of vascular anomalies associated with ICH, vascular neurosurgeons today require a thorough understanding of the various intraoperative imaging modalities available for obtaining real-time information. Use of one or more of these modalities may improve the surgeon's confidence during the procedure, the patient's safety during surgery, and surgical outcome.

The modern techniques discussed include 1) indocyanine green-based video angiography, which provides realtime information based on high-quality images showing the residual filling of vascular pathological entities and the patency of blood vessels of any size in the surgical field; and 2) intraoperative angiography, which remains the gold standard intraoperative diagnostic test in the surgical management of cerebral aneurysms and arteriovenous malformations. Hybrid procedures, providing multimodality image-guided surgeries and combining endovascular with microsurgical strategies within the same surgical session, have become feasible and safe. Microdoppler is a safe, noninvasive, and reliable technique for evaluation of hemodynamics of vessels in the surgical field, with the advantage of ease of use. Intraoperative MRI provides an effective navigation tool for cavernoma surgery, in addition to assessing the extent of resection during the procedure. Intraoperative CT scanning has the advantage of very high sensitivity to acute bleeding, thereby assisting in the confirmation of the extent of hematoma evacuation and the extent of vascular anomaly resection. Intraoperative ultrasound aids navigation and evacuation assessment during intracerebral hematoma evacuation surgeries. It supports the concept of minimally invasive surgery and has undergone extensive development in recent years, with the quality of ultrasound imaging having improved considerably.

Image-guided therapy, combined with modern intraoperative imaging modalities, has changed the fundamentals of conventional vascular neurosurgery by presenting real-time visualization of both normal tissue and pathological entities. These imaging techniques are important adjuncts to the surgeon's standard surgical armamentarium. Familiarity with these imaging modalities may help the surgeon complete procedures with improved safety, efficiency, and clinical outcome.
\end{abstract}

(http://thejns.org/doi/abs/10.3171/2013.2.FOCUS1324)

KEY WORDS $\bullet \quad$ intracerebral hemorrhage $\bullet$ intraoperative imaging $\bullet$
indocyanine green $\bullet \quad$ angiography $\bullet$ magnetic resonance imaging $\bullet$ ultrasound

I NTRACEREBRAL hemorrhage occurs in many conditions and has a wide spectrum of causes; $;^{7,29,31,39,42,58}$ most commonly, ICH occurs as a result of degenerative vascular disease, amyloid angiopathy, and hemorrhagic transforma-

\footnotetext{
Abbreviations used in this paper: $\mathrm{AVM}=$ arteriovenous malformation; CTA = CT angiography; DSA = digital subtraction angiography; ICG-VA = indocyanine green-based video angiography; $\mathrm{ICH}=$ intracerebral hemorrhage; iCT = intraoperative CT; iDSA = intraoperative DSA; iMD = intraoperative midcrodoppler; iMRI = intraoperative MRI; iUS = intraoperative ultrasound.
}

tions of ischemic strokes. However, up to $10 \%$ of ICHs are due to underlying vascular structural anomalies (AVMs, aneurysms, and cavernous angiomas being the most common). Other less common vascular malformations include sinus or cortical vein thrombosis, arteriovenous fistula, and arterial dissection.

Intracerebral hemorrhage is associated with a high early mortality rate and a significant long-term morbidity rate. Among all stroke subtypes, it is considered to be the one with the highest mortality rate. Hematoma volume is an important predictor of 30-day mortality, and hema- 
toma growth is a principal cause of early neurological deterioration. ${ }^{6,10,47}$ One should especially emphasize the high percentage of vascular pathological entities associated with $\mathrm{ICH}$ in the pediatric population, in which such entities were confirmed in a recent series in $61 \%$ of pediatric patients. ${ }^{38}$

Therapy for ICH is directed at blood clot removal, reduction of intracranial pressure, and of secondary associated damage. In the International Surgical Trial in Intracerebral Haemorrhage (STICH), ${ }^{30}$ the effect of medical versus surgical management of ICH was compared, but, except for a small subgroup of patients who experienced a benefit from surgery, no significant advantage was demonstrated for any of the treatments.

The concept of "suction surgeries" (referring to ICH removal through large craniotomies) is now obsolete. Vascular neurosurgeons treating ICH should be prepared to deal not only with clot removal but also with the underlying cause. These tasks are often complex, both from the morphological and the technical point of view, and increase the risk for potential vessel compromise. For effective occlusion and safe obliteration of vascular anomalies, the neurosurgeon today may require intraoperative imaging modalities for obtaining real-time information. The use of intraoperative imaging modalities is of particular relevance when preoperative vascular imaging studies are negative but the intraoperative findings reveal a discrete lesion. In these complex cases the surgeon has to adapt and perform the procedure without a complete picture of the task ahead. Intraoperative imaging assessment in such situations can be particularly informative.

Along with the advances in neurosurgical techniques, significant progress has been made in the field of intraoperative imaging, especially intraoperative neurovascular imaging. From the development of uniplane angiography, the first description of CT contrast infusion to identify vascular lesions, ${ }^{33}$ and Doppler techniques ${ }^{35}$ to MR angiography ${ }^{48}$ and transcranial Doppler, ${ }^{1}$ reliable intraoperative imaging technologies have been developed. Several intraoperative modalities have been implemented: ICGVA, iDSA, iCT, iMRI, iMD, and 3D iUS. These new imaging techniques and the concept of true hybrid rooms providing multimodality image-guided surgeries have improved diagnostics considerably. Such techniques may significantly assist the surgeon in challenging cases.

This paper reviews the current intraoperative imaging tools and the clinical use of each modality. Our experience with the various modalities and their advantages and potential limitations are discussed.

\section{Intraoperative Imaging Techniques}

\section{Indocyanine Green-Based Video Angiography}

Indocyanine green-based video angiography provides real-time information on the residual filling of aneurysms and on the patency of blood vessels of any size in the surgical field, including perforators, based on high-quality images. First introduced into the neurosurgical operating room in $2003,{ }^{40,41}$ The ICG-VA technique was found to be a simple and safe way to assess blood flow intraoperatively. ${ }^{46}$ Sequential studies compared the findings of ICG-VA with iDSA or postoperative DSA and reported them to be comparable in $90 \%$ of cases. ${ }^{27}$ Subsequently, the use of DSA for intraoperative evaluation of aneurysms has decreased. ${ }^{15}$ In the setting of ICH surgery with an underlying vascular pathological entity such as cerebral aneurysm or AVM, ICG is a powerful tool (Fig. 1). The ICG-VA technique is now widely used in multiple settings beyond aneurysms. . $2,18,22-24,32,54^{\text {It }}$ is routinely used for intracranial and spinal $\mathrm{AVMs}^{22}$ and arteriovenous fistulas. ${ }^{23}$ Unquestionably, ICG-VA has become an important adjuvant in vascular neurosurgery.

However, ICG is not suited for all applications. Its use is limited to the microscope's field of view and only to exposed blood vessels, so that not all vasculature can be

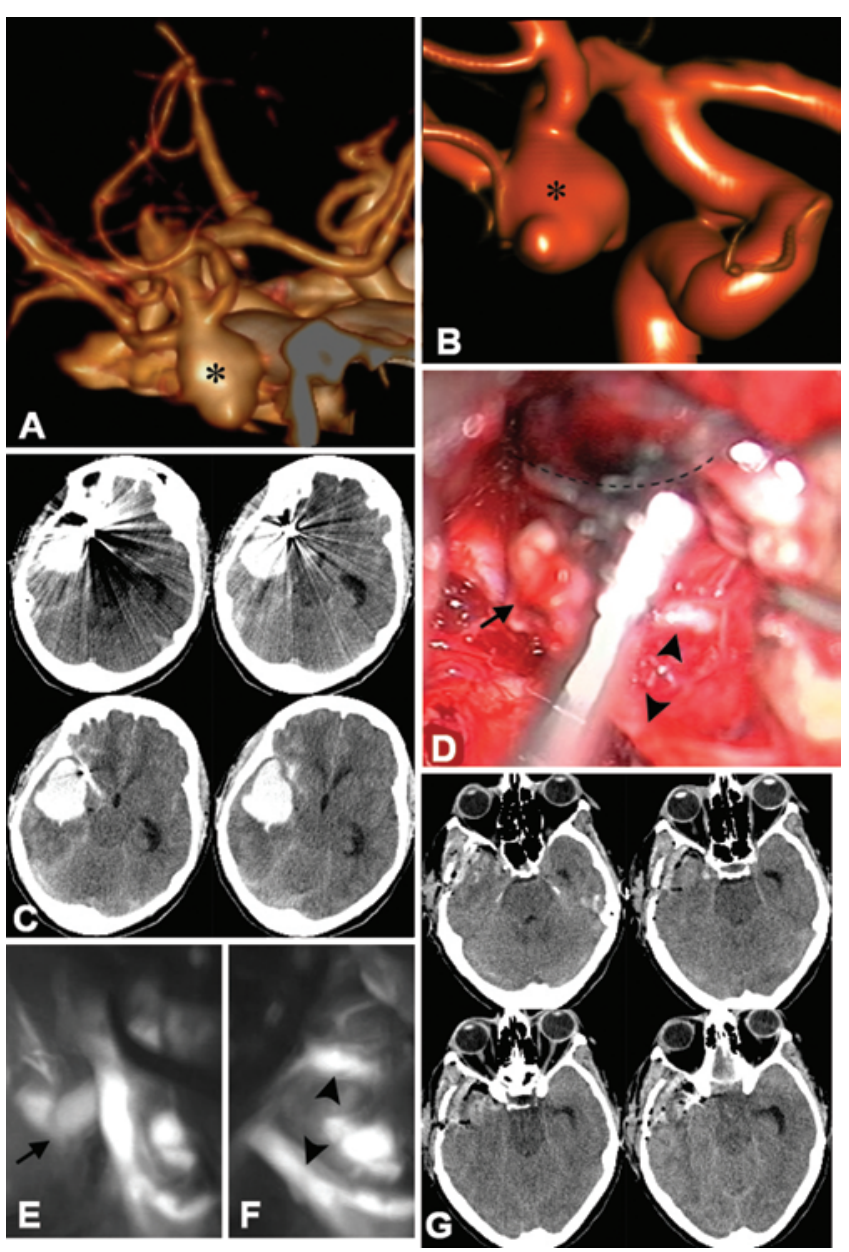

FIG. 1. A right MCA aneurysm (asterisks) is demonstrated on preoperative $3 \mathrm{D}$ reconstructions of CTA $(\mathrm{A})$ and on preoperative DSA reconstructions (B). An attempted endovascular closure of the aneurysm ended in aneurysm rupture and significant $\mathrm{ICH}$ (C; artifacts are caused by coils). The patient was urgently transported to the operating room and a right pterional approach was implemented. After evacuation of the $\mathrm{ICH}$, an optical mode photograph (D) demonstrating the right $\mathrm{M}_{1}$ branch (arrow) and the 2 right $\mathrm{M}_{2}$ branches (arrowheads) was obtained. The area of the coil-treated dome portion of the aneurysm is depicted by the dashed line. Intraoperative ICG-VA photographs showing patent circulation in the right $\mathrm{M}_{1}$ branch (E; arrow) and in the 2 right $\mathrm{M}_{2}$ branches (F; arrowheads). Postoperative CT scans (G) showing a satisfactory evacuation of the $\mathrm{ICH}$. 


\section{Intraoperative imaging modalities for intracerebral hemorrhage}

assessed. In addition, there are various factors that may limit the accuracy of ICG fluorescence signals, such as thick-walled or partially thrombosed aneurysms or cases of deep-seated or giant aneurysms. ${ }^{27}$ The utility of this imaging modality in the setting of AVM surgery is still unclear because of these visualization issues. Because only the parts of the AVM that are already visible in the surgical field will be visible to ICG-VA, standard intraoperative DSA remains the gold standard imaging modality in the assessment of AVM resection.

We use ICG-VA on a routine basis during vascular surgeries, including ICH evacuations, when the need arises (Fig. 2). It helps us assess residual filling of vascular lesions such as aneurysms and the patency of surrounding vessels after clipping. Sometimes multiple injections are performed after complex clip reconstructions. Use of ICG-VA has reduced our need for intraoperative DSA, which is now reserved for more difficult cases or those in which there is doubt following the ICG-VA.

\section{Intraoperative DSA}

This is a powerful diagnostic tool in the surgical management of cerebral aneurysms and AVMs and is still considered the gold standard procedure when assessing precise aneurysm clipping and vessel patency. It allows the neurosurgeon to confirm complete occlusion of the aneurysm and to demonstrate patency of all surrounding vasculature.

Several groups have evaluated iDSA efficiency and have revealed that it is both effective and accurate. , $^{97,49}$ In the largest single-center study, of 1093 vascular cases in which patients underwent routine iDSA, a greater than $8 \%$ revision of clip placement due to abnormalities found on iDSA was reported. ${ }^{9}$ In 9 (8.9\%) of 101 patients, iDSA demonstrated residual AVM requiring additional resection. Two of these patients $(22.2 \%)$ required a second surgical revision, and successful excision of the residual AVM was confirmed by repeat iDSA in all 9 patients. With minimal risk of morbidity $(0.99 \%)$ and neurological complications occurring in only $0.09 \%$ of cases, the authors reaffirmed iDSA safety and utility in the surgical treatment of aneurysms and vascular malformations.

As always, the value of iDSA must be weighed against the risk of complications and technical difficulties. ${ }^{14,21}$ The reported complication rate is as high as $3 \%$. Various technical challenges include the need for skilled neuroradiology staff, high costs, the need for a DSA C-arm, a radiolucent operating table head holder, and angiography equipment. At times the additional equipment can make the operating room particularly cramped (Fig. 3). In the
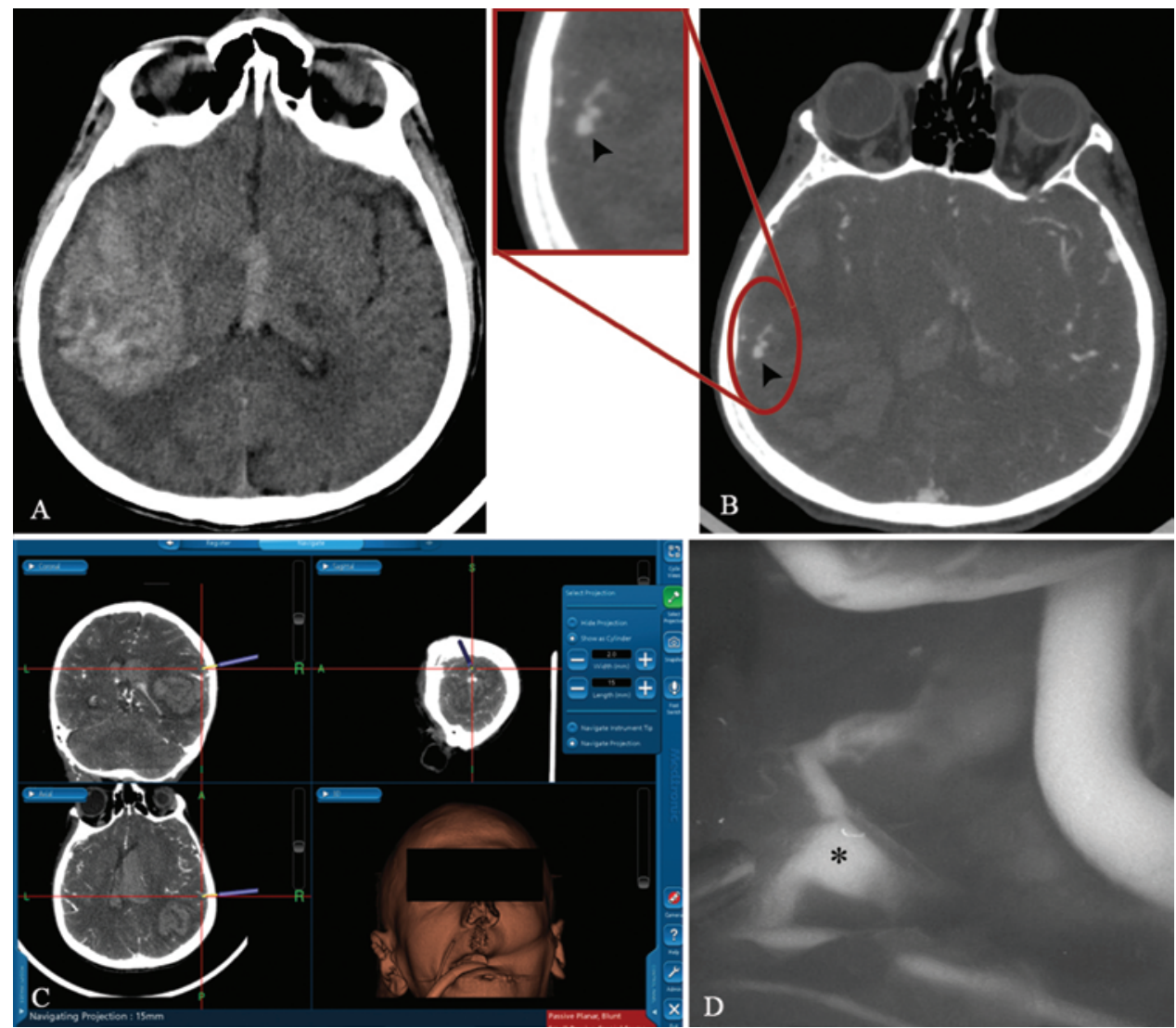

FIG. 2. Imaging studies obtained in a 20 -year-old man under treatment for subacute bacterial endocarditis who suddenly developed headache and left hemiparesis and subsequently lost consciousness. A: Preoperative CT scan showing right temporooccipital ICH involving the ventricular system. B: Preoperative CTA scan demonstrating a right distal sylvian fissure mycotic aneurysm (see inset, arrowhead). C: Preoperative neuronavigation images obtained using the Stealth S7 system for craniotomy planning. D: Intraoperative ICG-VA photograph locating the mycotic aneurysm (asterisk) and differentiating it from the parent artery. 


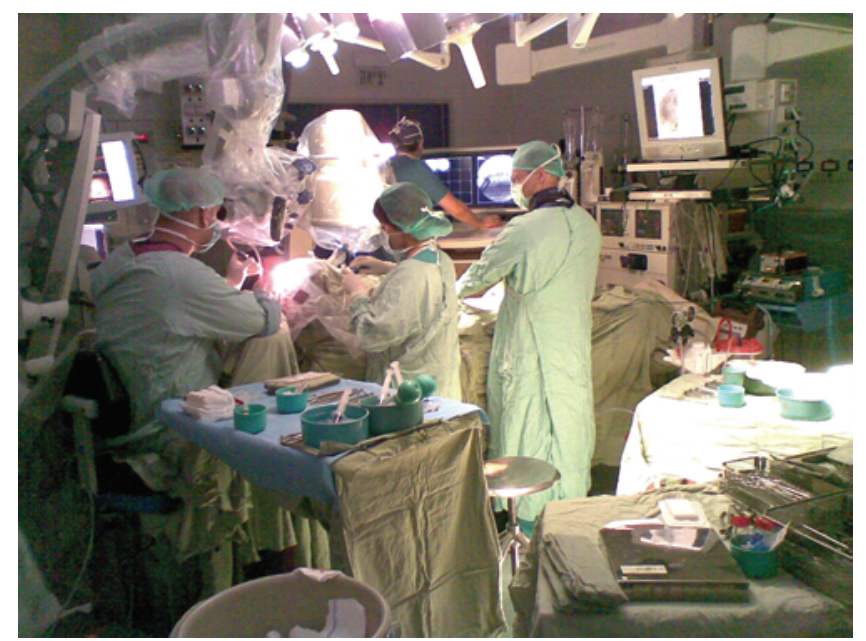

FIG. 3. Photograph showing the operating room setup for microsurgical clipping of an MCA aneurysm with iDSA guidance. Although this is still considered the gold standard procedure when assessing precise aneurysm clipping and vessel patency, one should consider the various technical difficulties involved.

setting of ICH treatment, iDSA may be too cumbersome to perform during an urgent operation due to availability of staff or equipment.

The drawbacks of iDSA may lead some to conclude that it is not a practical tool for all vascular cases. It is therefore best applied selectively, for complex vascular malformations and lesions in challenging anatomical locations.

\section{Hybrid Operating Room: Applications in ICH Surgery}

The concept of a hybrid operating room combining endovascular and microsurgical strategies within the same surgical session is applicable, cost-effective, and safe..$^{11,19,20,25}$ Full biplane neuroangiography in a fully equipped neurosurgical operating room provides a seamless transition between the operation and iDSA, which can be performed without repositioning the patient or moving in a portable $\mathrm{C}$-arm. The result is higher-quality angiography in 2 planes, which provides immediate intraoperative assessment of vessel patency and occlusion rate of different vascular pathological entities.

An example for this kind of setup is the addition of an iMRI unit to the hybrid operating room. Examples are the Advanced Multimodality Image-Guided Operating (A.M.I.G.O.) Suite at the Brigham and Women's Hospital and Harvard Medical School ${ }^{20}$ and the implementation of so-called IMRIS suites (IMRIS, Inc.) at various locations internationally. ${ }^{25}$ These systems are able to integrate a 1.5- to 3-T iMRI unit into a fully operational neurosurgical operating room and a full biplane angiography suite.

\section{Intraoperative Microdoppler Techniques}

Microdoppler imaging is a safe, noninvasive, and reliable technique for evaluation of the hemodynamics of vessels in the surgical field and of vascular malformations. It is low cost, and the time to result is $1-5$ minutes in most cases. Additionally, it shows good correlation with postoperative angiography and is therefore widely used. ${ }^{45}$ It allows for the measurement of blood flow velocities in the malformation itself and in the surrounding vasculature. It is a valuable tool for providing immediate feedback to the surgeon, thereby improving the chances for an optimal surgical outcome because intraoperative complications such as vessel occlusion can be diagnosed and dealt with immediately. Several groups compared iMD findings to those in iDSA or postoperative DSA. ${ }^{4,5,13,28,45,55}$ They found a high rate of concordance between the $\mathrm{MMD}$ and angiographic findings regarding proper clip placement and complete occlusion of an intracranial aneurysm and associated clip-induced adjacent-vessel stenosis.

There are several iMD techniques available on the market today. Some are simple and cost-efficient, whereas others, such as the ultrasonic perivascular flow probe, are more advanced but are expensive and more cumbersome. ${ }^{4}$ The iMD modality carries the advantage of speed and ease of use and is therefore very attractive during surgery for ICH. The primary disadvantage of this technique is its inability to identify residual aneurysm remnants in cases of thrombosed or low-flow aneurysms-which are readily identified by intraoperative angiography and ICG-VA. That is to say, iMD provides qualitative and not quantitative results to the surgeon. Additionally, it is only useful for high-caliber vessels observed under the operating microscope.

\section{Intraoperative MRI}

Intraoperative MRI has been incorporated into modern neurosurgical operating rooms for more than a decade as a guide for neurosurgical interventions. ${ }^{3,34,43,44}$ This technology has proved to be a useful modality in vascular neurosurgery, especially for cavernous angiomas and AVMs. ${ }^{43}$ It provides a highly accurate and precise navigation tool, with excellent resolution, which is a prerequisite for localizing and targeting vascular lesions. It also addresses the problem of the ever-changing organization of intracranial structures during surgery by providing near-real-time, high-quality images.

One of the drawbacks of intraoperative MRI with regard to resection of vascular lesions is delineating the extent of resection. Clearly determining the edge of the lesion can be challenging due to the deposition of hemosiderin from hemorrhage. There are several types of MRI units used today. ${ }^{44}$ In our experience the use of iMRI in ICH surgery is limited to cavernoma surgery, and in cases in which MRI may help in delineating the margins of deep lesions surrounded with hematoma, in those cases we consider the low-field portable systems as the most useful. An example of a low-field iMRI unit is the OdinPoleStar system, which was first introduced in 2001.17 This compact MR scanner can be installed in a standard operating theater without major modification (Fig. 4). It functions with both an integrated optical system and an MRI tracking system and is operated by the neurosurgeon from an in-room computer workstation. ${ }^{16,36}$

\section{Intraoperative $C T$}

Intraoperative CT imaging is a standard tool in the successful planning and execution of a diverse range of 

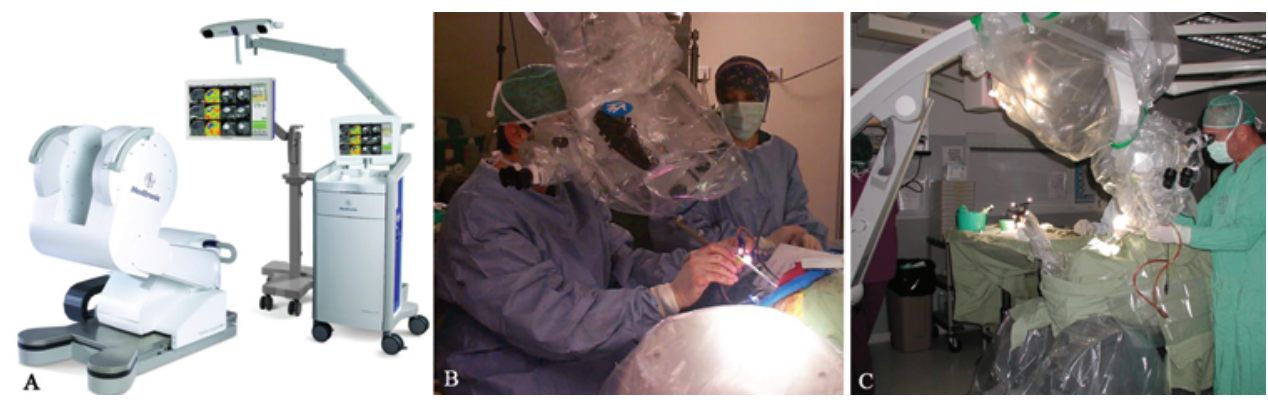

FIG. 4. Photograph showing the compact and mobile Medtronic PoleStar N30 surgical MRI system, consisting of a 0.15-T MRI unit integrated with a Medtronic StealthStation navigation system (A). Photographs showing the system being used for craniotomy approach for an ICH surgery related to cerebral cavernous angiomas (B), and for a transsphenoidal approach in a case of pituitary apoplexy (C). Reprinted with permission from Medtronic, Inc.

neurosurgical procedures. ${ }^{8}$ In the treatment of ICH, iCT systems such as the CereTom mobile scanner are used to assist in the confirmation of intraoperative positioning of catheters, the extent of hematoma evacuation, and the extent of vascular anomaly resection. The iCT images can be obtained and merged with those obtained using other modalities such as MRI if desired. Limitations of iCT include lower resolution than the large CT scanners, technician availability, and intraoperative imaging of the skull base. In the setting of ICH surgery, iCT has the advantage of very high sensitivity to acute blood. ${ }^{2,50}$

\section{Ultrasound-Guided Evacuation of ICH}

The iUS technique has undergone extensive development in recent years, and the quality of ultrasound imaging has improved considerably. ${ }^{26,51-53,55-57}$ Still, many neurosurgeons have been reluctant to try advanced versions of iUS due to negative past experiences. The quality of iUS imaging has improved significantly, such that the surgeon can readily assess ICH dimensions and identify structures within the brain in real time. This modality supports the concept of minimally invasive surgery by allowing the surgeon to identify which part of the clot presents closer to the cortical surface, thereby minimizing disruption to the surrounding brain because the shortest transcortical trajectory is taken to enter the clot. Orientation problems of iUS were improved by integrating navigation technologies. As a result, neurosurgeons find it relatively easy to understand and use the iUS navigation systems found in the market today. ${ }^{53}$

The integration of 3D iUS and neuronavigation technology has created a real-time imaging method that can accurately demonstrate ICH. The 3D iUS systems such as the SonoWand have made this technology an efficient tool that can combine the real-time feedback from iUS with the detailed preoperative imaging from CT or MRI studies. During ICH evacuation procedures, iUS facilitates directing an instrument such as a Cavitron ultrasonic surgical aspirator or an endoscope to a target point in real time. During various vascular operations the iUS helps localize lesions and characterize their internal structure, and distance from the surface to the target can be calculated. ${ }^{57,58}$ The relation of various lesions to the surrounding brain can be appreciated before, during, and after excision of vascular pathological entities.

\section{Conclusions}

There have been remarkable advances in the intraoperative imaging techniques used in the surgical management of ICH. They have enabled neurosurgeons to visualize and delineate anatomical and pathological structures more accurately, even during the surgery itself. Image-guided therapy has changed the fundamentals of conventional surgery by presenting accurate visualization of both normal tissue and lesion. Particularly within the last decade, advanced imaging modalities have become standard tools of neurosurgical practice, creating image-guided surgery as a subspecialty. In discussing the potential advantages and disadvantages of each modality, it should be pointed out that they are not mutually exclusive. On the contrary, the various intraoperative imaging modalities used in the surgery for ICH may be complementary, and therefore vascular neurosurgeons should be prepared to use any intraoperative imaging modality when the need arises.

\section{Disclosure}

The authors report no conflict of interest concerning the materials or methods used in this study or the findings specified in this paper.

Author contributions to the study and manuscript preparation include the following. Conception and design: Harnof, Goren. Acquisition of data: Goren, Monteith. Analysis and interpretation of data: Monteith, Bakon. Drafting the article: Harnof, Goren, Monteith. Critically revising the article: Monteith. Administrative/technical/ material support: Harnof, Goren. Study supervision: Hadani, Bakon.

\section{References}

1. Aaslid R, Huber P, Nornes H: Evaluation of cerebrovascular spasm with transcranial Doppler ultrasound. J Neurosurg 60: 37-41, 1984

2. Agrawal D, Sahoo S, Satyarthee GD, Gupta D, Sinha S, Misra MC: Initial experience with mobile computed tomogram in neurosurgery intensive care unit in a level 1 trauma center in India. Neurol India 59:739-742, 2011

3. Albayrak B, Samdani AF, Black PM: Intra-operative magnetic resonance imaging in neurosurgery. Acta Neurochir (Wien) 146:543- 557, 2004

4. Amin-Hanjani S, Meglio G, Gatto R, Bauer A, Charbel FT: The utility of intraoperative blood flow measurement during aneurysm surgery using an ultrasonic perivascular flow probe. Neurosurgery 62 (6 Suppl 3):1346-1353, 2008

5. Bailes JE, Tantuwaya LS, Fukushima T, Schurman GW, Davis 


\section{O. Goren et al.}

D: Intraoperative microvascular Doppler sonography in aneurysm surgery. Neurosurgery 40:965-972, 1997

6. Broderick JP, Brott TG, Duldner JE, Tomsick T, Huster G: Volume of intracerebral hemorrhage. A powerful and easy-touse predictor of 30-day mortality. Stroke 24:987-993, 1993

7. Butcher K, Laidlaw J: Current intracerebral haemorrhage management. J Clin Neurosci 10:158-167, 2003

8. Carlson AP, Yonas H: Portable head computed tomography scanner-technology and applications: experience with 3421 scans. J Neuroimaging 22:408-415, 2012

9. Chalouhi N, Theofanis T, Jabbour P, Dumont AS, Fernando Gonzalez L, Starke RM, et al: Safety and efficacy of intraoperative angiography in craniotomies for cerebral aneurysms and arteriovenous malformations: a review of 1093 consecutive cases. Neurosurgery 71:1162-1169, 2012

10. Davis SM, Broderick J, Hennerici M, Brun NC, Diringer MN, Mayer SA, et al: Hematoma growth is a determinant of mortality and poor outcome after intracerebral hemorrhage. Neurology 66:1175-1181, 2006

11. Fandino J, Taussky P, Marbacher S, Muroi C, Diepers M, Fathi AR, et al: The concept of a hybrid operating room: applications in cerebrovascular surgery. Acta Neurochir Suppl 115: 113-117, 2013

12. Ferroli P, Acerbi F, Albanese E, Tringali G, Broggi M, Franzini A, et al: Application of intraoperative indocyanine green angiography for CNS tumors: results on the first 100 cases. Acta Neurochir Suppl 109:251-257, 2011

13. Firsching R, Synowitz HJ, Hanebeck J: Practicability of intraoperative microvascular Doppler sonography in aneurysm surgery. Minim Invasive Neurosurg 43:144-148, 2000

14. Friedman JA, Kumar R: Intraoperative angiography should be standard in cerebral aneurysm surgery. BMC Surg 9:7, 2009

15. Gruber A, Dorfer C, Standhardt H, Bavinzski G, Knosp E: Prospective comparison of intraoperative vascular monitoring technologies during cerebral aneurysm surgery. Neurosurgery 68:657-673, 2011

16. Hadani M: Development and design of low field compact intraoperative MRI for standard operating room. Acta Neurochir Suppl 109:29-33, 2011

17. Hadani M, Spiegelman R, Feldman Z, Berkenstadt H, Ram Z: Novel, compact, intraoperative magnetic resonance imaging-guided system for conventional neurosurgical operating rooms. Neurosurgery 48:799-809, 2001

18. Haga S, Nagata S, Uka A, Akagi Y, Hamada Y, Shono T: Near-infrared indocyanine green videoangiography for assessment of carotid endarterectomy. Acta Neurochir (Wien) 153:1641-1644, 2011

19. Iihara K, Satow T, Matsushige T, Kataoka H, Nakajima N, Fukuda K, et al: Hybrid operating room for the treatment of complex neurovascular and brachiocephalic lesions. J Stroke Cerebrovasc Dis [epub ahead of print], 2012

20. Jolesz FA: Intraoperative imaging in neurosurgery: where will the future take us? Acta Neurochir Suppl 109:21-25, 2011

21. Katz JM, Gologorsky Y, Tsiouris AJ, Wells-Roth D, Mascitelli $\mathrm{J}$, Gobin YP, et al: Is routine intraoperative angiography in the surgical treatment of cerebral aneurysms justified? A consecutive series of 147 aneurysms. Neurosurgery 58:719-727, 2006

22. Killory BD, Nakaji P, Gonzales LF, Ponce FA, Wait SD, Spetzler RF: Prospective evaluation of surgical microscope-integrated intraoperative near-infrared indocyanine green angiography during cerebral arteriovenous malformation surgery. Neurosurgery 65:456-462, 2009

23. Killory BD, Nakaji P, Maughan PH, Wait SD, Spetzler RF: Evaluation of angiographically occult spinal dural arteriovenous fistulae with surgical microscope-integrated intraoperative near-infrared indocyanine green angiography: report of 3 cases. Neurosurgery 68:781-787, 2011

24. Laakso A, Hernesniemi J, Yonekawa Y, Tsukahara T (eds):
Surgical Management of Cerebrovascular Disease. Vienna: Springer, 2010

25. Lang MJ, Greer AD, Sutherland GR: Intra-operative MRI at 3.0 Tesla: a moveable magnet. Acta Neurochir Suppl 109: $151-156,2011$

26. Lee JK, Lee JH: Ultrasound-guided evacuation of spontaneous intracerebral hematoma in the basal ganglia. J Clin Neurosci 12:553-556, 2005

27. Li J, Lan Z, He M, You C: Assessment of microscope-integrated indocyanine green angiography during intracranial aneurysm surgery: a retrospective study of 120 patients. Neurol India 57:453-459, 2009

28. Marchese E, Albanese A, Denaro L, Vignati A, Fernandez E, Maira G: Intraoperative microvascular Doppler in intracranial aneurysm surgery. Surg Neurol 63:336-342, 2005

29. Mayer SA, Rincon F: Treatment of intracerebral haemorrhage. Lancet Neurol 4:662-672, 2005

30. Mendelow AD, Gregson BA, Fernandes HM, Murray GD, Teasdale GM, Hope DT, et al: Early surgery versus initial conservative treatment in patients with spontaneous supratentorial intracerebral haematomas in the International Surgical Trial in Intracerebral Haemorrhage (STICH): a randomised trial. Lancet 365:387-397, 2005

31. Morgenstern LB, Hemphill JC III, Anderson C, Becker K, Broderick JP, Connolly ES Jr, et al: Guidelines for the management of spontaneous intracerebral hemorrhage: a guideline for healthcare professionals from the American Heart Association/ American Stroke Association. Stroke 41:2108-2129, 2010

32. Murai Y, Adachi K, Matano F, Tateyama K, Teramoto A: Indocyanin green videoangiography study of hemangioblastomas. Can J Neurol Sci 38:41-47, 2011

33. Newell DW, LeRoux PD, Dacey RG Jr, Stimac GK, Winn HR: CT infusion scanning for the detection of cerebral aneurysms. J Neurosurg 71:175-179, 1989

34. Nimsky C, Ganslandt O, Von Keller B, Romstöck J, Fahlbusch R: Intraoperative high-field-strength MR imaging: implementation and experience in 200 patients. Radiology 233:67-78, 2004

35. Nornes H, Grip A, Wikeby P: Intraoperative evaluation of cerebral hemodynamics using directional Doppler technique. Part 2: Saccular aneurysms. J Neurosurg 50:570-577, 1979

36. Ntoukas V, Krishnan R, Seifert V: The new generation polestar $\mathrm{n} 20$ for conventional neurosurgical operating rooms: a preliminary report. Neurosurgery 62 (3 Suppl 1):82-90, 2008

37. Pai BS, Muralimohan S: Intraoperative angiography in aneurysm surgery: an initial experience. Neurol India 58:571-575, 2010

38. Papadias A, Taha A, Sgouros S, Walsh AR, Hockley AD: Incidence of vascular malformations in spontaneous intra-cerebral haemorrhage in children. Childs Nerv Syst 23:881-886, 2007

39. Qureshi AI, Tuhrim S, Broderick JP, Batjer HH, Hondo H, Hanley DF: Spontaneous intracerebral hemorrhage. N Engl J Med 344:1450-1460, 2001

40. Raabe A, Beck J, Gerlach R, Zimmermann M, Seifert V: Nearinfrared indocyanine green video angiography: a new method for intraoperative assessment of vascular flow. Neurosurgery 52:132-139, 2003

41. Raabe A, Nakaji P, Beck J, Kim LJ, Hsu FP, Kamerman JD, et al: Prospective evaluation of surgical microscope-integrated intraoperative near-infrared indocyanine green videoangiography during aneurysm surgery. J Neurosurg 103:982-989, 2005

42. Sacco S, Marini C, Toni D, Olivieri L, Carolei A: Incidence and 10-year survival of intracerebral hemorrhage in a population-based registry. Stroke 40:394-399, 2009

43. Schwartz RB, Hsu L, Wong TZ, Kacher DF, Zamani AA, Black PM, et al: Intraoperative MR imaging guidance for intracranial neurosurgery: experience with the first 200 cases. Radiology 211:477-488, 1999 


\section{Intraoperative imaging modalities for intracerebral hemorrhage}

44. Seifert V: Intraoperative MRI in neurosurgery: technical overkill or the future of brain surgery? Neurol India 51:329-332, 2003

45. Siasios I, Kapsalaki EZ, Fountas KN: The role of intraoperative micro-Doppler ultrasound in verifying proper clip placement in intracranial aneurysm surgery. Neuroradiology 54: 1109-1118, 2012

46. Snyder LA, Spetzler RF: Current indications for indocyanine green angiography. World Neurosurg 76:405-406, 2011

47. Steiner T, Bösel J: Options to restrict hematoma expansion after spontaneous intracerebral hemorrhage. Stroke 41:402409, 2010

48. Sutherland GR, Kaibara T, Louw D, Hoult DI, Tomanek B, Saunders J: A mobile high-field magnetic resonance system for neurosurgery. J Neurosurg 91:804-813, 1999

49. Tang G, Cawley CM, Dion JE, Barrow DL: Intraoperative angiography during aneurysm surgery: a prospective evaluation of efficacy. J Neurosurg 96:993-999, 2002

50. Uhl E, Zausinger S, Morhard D, Heigl T, Scheder B, Rachinger $\mathrm{W}$, et al: Intraoperative computed tomography with integrated navigation system in a multidisciplinary operating suite. Neurosurgery 64 (5 Suppl 2):231-240, 2009

51. Unsgaard G, Gronningsaeter A, Ommedal S, Nagelhus Hernes TA: Brain operations guided by real-time two-dimensional ultrasound: new possibilities as a result of improved image quality. Neurosurgery 51:402-412, 2002

52. Unsgaard G, Rygh OM, Selbekk T, Müller TB, Kolstad F, Lindseth F, et al: Intra-operative 3D ultrasound in neurosurgery. Acta Neurochir (Wien) 148:235-253, 2006

53. Unsgård G, Solheim O, Lindseth F, Selbekk T: Intra-operative imaging with 3D ultrasound in neurosurgery. Acta Neurochir Suppl 109:181-186, 2011

54. Wang JT, Zhang D, Zuo F, Wang S: [Application of indocyanine green angiography in bypass surgery for moyamoya disease.] Zhonghua Yi Xue Za Zhi 90:1628-1630, 2010 [Chinese]

55. Woydt M, Greiner K, Perez J, Krone A, Roosen K: Intraoperative color duplex sonography of basal arteries during aneurysm surgery. J Neuroimaging 7:203-207, 1997

56. Woydt M, Krone A, Soerensen N, Roosen K: Ultrasoundguided neuronavigation of deep-seated cavernous haemangiomas: clinical results and navigation techniques. Br J Neurosurg 15:485-495, 2001

57. Woydt M, Perez J, Meixensberger J, Krone A, Soerensen N, Roosen K: Intra-operative colour-duplex-sonography in the surgical management of cerebral AV-malformations. Acta Neurochir (Wien) 140:689-698, 1998

58. Zahuranec DB, Gonzales NR, Brown DL, Lisabeth LD, Longwell PJ, Eden SV, et al: Presentation of intracerebral haemorrhage in a community. J Neurol Neurosurg Psychiatry 77: $340-344,2006$

Manuscript submitted January 15, 2013.

Accepted February 15, 2013.

Please include this information when citing this paper: DOI: 10.3171/2013.2.FOCUS1324.

Address correspondence to: Sagi Harnof, M.D., Department of Neurosurgery, The Chaim Sheba Medical Center, Tel Hashomer 52621, Israel.email: sagi.harnof@sheba.health.gov.il. 